\section{Pharmacokinetic considerations in the treatment of hypertension in risperidone-medicated patients - thinking of clinically relevant CYP2D6 interactions}

\author{
Michael Paulzen ${ }^{1,2}$, Ekkehard Haen ${ }^{3,4}$, Gerhard Gründer ${ }^{1,2}$, \\ Sarah E Lammertz ${ }^{1,2}$, Benedikt Stegmann ${ }^{3,4}$, Koen RJ Schruers ${ }^{5}$, \\ Sebastian Walther ${ }^{6}$ and Georgios Schoretsanitis ${ }^{1,2,6}$
}

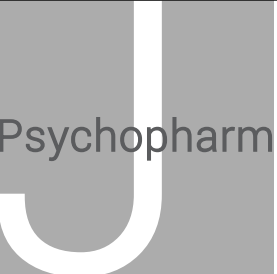

Journal of Psychopharmacology 2016, Vol. 30(8) 803-809 (c) The Author(s) 2016 Reprints and permissions: sagepub.co.uk/journalsPermissions.nav DOI: $10.1177 / 0269881116650390$ jop.sagepub.com

@SAGE

\begin{abstract}
Background: Treatment of arterial hypertension in patients with severe mental illnesses often results in polypharmacy, potentially leading to drug-drug interactions. The objective of the study was to analyse the in vivo inhibitory potential of two antihypertensive drugs, amlodipine and metoprolol on CYP2D6 catalysed 9-hydroxylation of risperidone (RIS).

Methods: A therapeutic drug monitoring database with plasma concentrations of RIS and 9-hydroxyrisperidone (9-0H-RIS) of 1584 patients was analysed. Three groups were considered; a group of patients receiving RIS without a potentially cytochrome influencing co-medication (control group, $\left.\mathrm{R}_{0}, n=852\right)$, a group co-medicated with amlodipine $\left(\mathrm{R}_{\mathrm{A}}, n=27\right)$ and a group, co-medicated with metoprolol $\left(\mathrm{R}_{\mathrm{M}}, n=41\right)$. Plasma concentrations, concentration-to-dose ratios (C/Ds) of RIS, 9-OH-RIS and the active moiety (AM), as well as the metabolic ratios were computed and compared using the Kruskal-Wallis test, the Mann-Whitney $U$ test and the Jonckheere-Terpstra test to determine the means and different patterns of distribution of plasma concentrations as well as the concentration-to-dose ratios.

Results: The median daily dosage of RIS did not differ between the groups $(p=0.708)$. No differences were found in median plasma concentrations of RIS, 9-OH-RIS and AM. However, concentration-to-dose ratios for RIS, 9-0H-RIS and AM were significantly higher in the amlodipine group ( $p=0.025$, $p=0.048$ and $p=0.005$ ). In the metoprolol group, the concentration-to-dose ratio for RIS was significantly higher than in the control group ( $p=0.017$ ), while the C/D for 9-0H-RIS and AM was not.

Conclusions and limitations: Our data show a potential pharmacokinetic interaction, most likely via CYP3A4 between amlodipine and RIS, reflected in significantly different C/Ds for RIS, 9-OH-RIS and AM. Although the interaction did not result in significantly higher plasma levels, changes in C/Ds and their distribution with regard to the median concentrations were observed.
\end{abstract}

Keywords

Therapeutic drug monitoring, risperidone, amlodipine, metoprolol, CYP2D6, interaction, pharmacokinetics

\section{Introduction}

A marked prevalence of cardiovascular diseases including arterial hypertension has been frequently reported in patients with severe mental illnesses such as schizophrenia (Bresee et al., 2010; Liao et al., 2011; Nasrallah et al., 2015). The high prevalence is followed by a concomitant need for non-pharmacological interventions and pharmacological treatment to prevent the patients from negative consequences (Hennekens et al., 2005). Some researchers reported an association between arterial hypertension, metabolic syndrome conditions and negative symptoms of schizophrenia (Sicras-Mainar et al., 2015). A plausible interpretation attributes the underlying sedentary lifestyle and lack of physical exercise to the negative symptoms, a key element of schizophrenia (Fervaha et al., 2014; Rabinowitz et al., 2012). Furthermore, antipsychotic treatment is considered to contribute to the relative risk of developing cardiovascular disease in schizophrenia. In contrast to first-generation antipsychotic agents (FGAs), second-generation antipsychotics (SGAs) are regarded as less cardiotoxic, but a chronic dopamine $\mathrm{D}_{2}$-receptor blockade by an SGA might lead to increased sympathetic tone and consequently to hypertension and an increased risk of cardiac arrhythmia by abolishing peripheral dopaminergic modulation

\footnotetext{
${ }^{1}$ Department of Psychiatry, Psychotherapy and Psychosomatics, RWTH Aachen University, Aachen, Germany

2Jülich Aachen Research Alliance, Translational Brain Medicine, Jülich/Aachen, Germany

${ }^{3}$ Department of Psychiatry and Psychotherapy, University of Regensburg, Regensburg, Germany

4Department of Pharmacology and Toxicology, University of Regensburg, Regensburg, Germany

${ }^{5}$ School for Mental Health and Neuroscience, Maastricht University, Maastricht, the Netherlands

${ }^{6}$ University Hospital of Psychiatry, Bern, Switzerland
}

\section{Corresponding author:}

Michael Paulzen, Department of Psychiatry, Psychotherapy and Psychosomatics and JARA - Translational Brain Medicine, RWTH Aachen University, Pauwelsstr. 30, 52074 Aachen, Germany. Email:mpaulzen@ukaachen.de 
(Scigliano and Ronchetti, 2013). An indirect mechanism may be based upon a drug-induced alteration of metabolic homeostasis (Correll et al., 2011) and weight gain may play a mediator role in schizophrenia-related hypertension (Goff et al., 2005; Nasrallah et al., 2006). Non-pharmacological interventions as well as pharmacological treatment of metabolic syndrome conditions and especially hypertension in patients with schizophrenia may be essential in eliminating the excess mortality rate and improvement of life expectancy.

However, the pharmacological treatment of hypertension in patients with severe mental illnesses often results in multiple drug therapy. Hence, the risk of adverse drug reactions (ADRs) and the potential for drug-drug interactions (DDIs) increases exponentially (Cadieux, 1989) with an increasing number of applied drugs. Therefore predicting a potential DDI is important for the safety and tolerability of pharmacotherapy in the clinical setting. Inhibition of cytochrome (CYP) metabolism is recognized as one of the most important mechanisms of clinical DDIs and may result in serious toxicological consequences, if dosage of the substrate is not adjusted (Hiemke et al., 2011). Most of the psychotropic drugs are metabolized extensively via CYP enzymes (Stingl et al., 2013) and some somatic drugs including quinidine, terbinafine or amiodarone, which are classified as strong, moderate or weak inhibitors of CYP2D6 according to the US Food and Drug Administration classification of in vivo inhibitors of CYP2D6 (US Food and Drug Administration, 2014) have the potential to cause clinically relevant DDIs. Recently, CYPmediated DDI and the understanding of pharmacokinetic DDI involving CYP isoenzymes have been acknowledged and several tables of drugs as substrates, inhibitors and inducers of drugmetabolizing CYP enzymes have been published (Hiemke et al., 2011; US Food and Drug Administration, 2014). However, any assessment regarding potential DDIs remains provisional considering the great number of drugs that have not been investigated yet with regard to cytochrome blocking or inducing properties.

Therapeutic drug monitoring (TDM) databases are a valuable source for a better understanding of potential pharmacokinetic interactions. Here, we investigated whether the antihypertensive drugs amlodipine or metoprolol have potential interactions with the CYP2D6-mediated risperidone (RIS) metabolism.

RIS, a benzisoxazole derivative, is a second generation antipsychotic (SGA) with selective antagonistic properties at receptors serotonin/hydroxytryptamine, $\mathrm{HT}, 5-\mathrm{HT}_{2}$ and dopamine $\left(\mathrm{D}_{2}\right)$ (Janssen et al., 1988). RIS has been used effectively in the treatment of schizophrenia and a broad spectrum of other psychiatric diseases over the past two decades with a low incidence of extrapyramidal symptoms (EPSs) and a lack of anticholinergic effects (Chouinard and Arnott, 1993; Leucht et al., 1999; Marder et al., 1997). The primary pathway of RIS metabolism is a CYP2D6-catalysed 9-hydroxylation and the main active metabolite is 9-hydroxyrisperidone (9-OH-RIS). Minor metabolic pathways include an oxidative N-dealkylation and 7-hydroxylation. RIS and its metabolites are eliminated via the urine and, to a much lesser extent, via the faeces. In vitro findings have revealed that CYP3A4 and CYP3A5 might also be involved in the 9-hydoxylation of RIS (Fang et al., 1999; Xiang et al., 2010; Yasui-Furukori et al., 2001). As 9-OH-RIS is pharmacologically active, clinicians consider the combined concentration of RIS and 9-OH-RIS (active moiety (AM)) as the most relevant measure. According to the Arbeitsgemeinschaft für Neuropsychopharmakologie und Pharmakopsychiatrie (AGNP) consensus guidelines, a so-called therapeutic reference range of $20-60 \mathrm{ng} / \mathrm{mL}$ is suggested for the AM (Hiemke et al., 2011).

Amlodipine is a long-acting dihydropyridine calcium-channel blocker (CCB) acting primarily as a peripheral vasodilator (Faulkner et al., 1986). It is slowly absorbed and extensively metabolized in the liver, mainly by CYP3A4 (Kim et al., 2006). Amlodipine seems to have an enhanced safety profile in patients with concomitant diabetes (Fukao et al., 2011) and there is some evidence supporting cardioprotective effects (Kjeldsen et al., 2014; Lee et al., 2014). To date, there is no clear evidence for CYP inhibiting or inducing properties of amlodipine resulting in increased or decreased drug concentrations of concomitantly applied CYP-metabolized drugs except some data about inhibitory effects on CYP3A4 mediated metabolism of the lipid-altering 3-hydroxy-3-methyl-glutaryl-coenzyme A reductase (HMG-CoA) reductase inhibitor simvastatin (Son et al., 2014; US Food and Drug Administration, 2014).

Metoprolol is a cardioselective beta-1-adrenoreceptor blocker without intrinsic sympathomimetic activity. Approximately 70 $80 \%$ of oral metoprolol is metabolized by CYP2D6 in the liver ( $\mathrm{Li}$ and Wang, 2006). A very recent study by Hefner et al. investigated a potential inhibitory effect of metoprolol on the CYP-mediated metabolism of venlafaxine (VEN) but found no effect on CYP2D6-catalysed formation of O-desmethylvenlafaxine (ODV). The lack of differences in plasma concentrations of VEN and ODV between the metoprolol group and the control group makes it unlikely that metoprolol could have a pharmacokinetic interaction potential via other CYP isoenzymes that are involved in VEN metabolism such as CYP3A4 or CYP 2C19, (Hefner et al., 2015).

As data on the inhibitory effects of amlodipine on CYP2D6 and/or CYP3A4/5 with regard to psychotropic drugs are missing and data about the effects of metoprolol remain inconsistent, we analysed data from a TDM survey obtained from patients whose antipsychotic treatment with RIS was individually optimized using TDM to further investigate potential effects of amlodipine and metoprolol on CYP2D6 mediated hydroxylation of RIS.

\section{Materials and methods}

The study was conducted as cooperation between the Department of Psychiatry, Psychotherapy and Psychosomatics of RWTH Aachen University Hospital, Aachen, Germany, and the Department of Psychiatry and Psychotherapy at the University of Regensburg, Germany. A large TDM database containing plasma concentrations of RIS and 9-OH-RIS of 1584 patients with a broad spectrum of psychiatric diseases (exception: organic mental disorders) was analysed. Data collection took place between 2006-2015 as part of the clinical routine in different institutions as part of the Arbeitsgemeinschaft Arzneimittelsicherheit bei psychischen Erkrankungen (AGATE), a cooperation for drug safety in the treatment of psychiatric diseases (for details see www.amuep-agate.de). The database consists of 1584 samples from in- and outpatients who had been treated with RIS. Retrospective analysis of clinical data for this study was in accordance with the local regulatory authority.

We considered three groups in this naturalistic database; a group of adult patients that received RIS as an oral formulation without a potentially CYP influencing co-medication (control group, $\mathrm{R}_{0}$ ) (Hiemke et al., 2011; US Food and Drug Administration, 2014), a group that was co-medicated with amlodipine $\left(\mathrm{R}_{\mathrm{A}}\right)$ and a third group that was co-medicated with 
Table 1. Patients' demographic characteristics.

\begin{tabular}{|c|c|c|c|c|c|}
\hline \multirow[t]{2}{*}{ Group } & \multirow[t]{2}{*}{ Number } & \multirow[t]{2}{*}{ Age (years) } & \multicolumn{2}{|l|}{ Gender } & \multirow{2}{*}{$\frac{\text { DD RIS (mg/day) }}{\text { Median (range) }}$} \\
\hline & & & $\%$ Females & $\%$ Males & \\
\hline Amlodipine $\left(R_{A}\right)$ & 27 & $61.0(27-80)^{a}$ & $74.1^{b}$ & 25.9 & $4.0(1.00-8.0)$ \\
\hline Metoprolol $\left(R_{M}\right)$ & 41 & $49.8(21-87)^{\mathrm{a}}$ & $36.6^{b}$ & 63.4 & $4.0(1.00-8.0)$ \\
\hline Control $\left(\mathrm{R}_{0}\right)$ & 852 & $41.25(18-87)^{a}$ & $44.2^{b}$ & 55.8 & $4.0(1.00-10.0)$ \\
\hline
\end{tabular}

DD: daily dosage; RIS: risperidone.

${ }^{a}$ Age values in the $R_{A}$ group were significantly higher than in the $R_{M}$ and the control group ( $p<0.001$ for Kruskal-Wallis test).

${ }^{b} G$ ender distributions differed between groups, with female patients being more in the $\mathrm{R}_{\mathrm{A}}$ group than in the control group and $\mathrm{R}_{\mathrm{M}}(p=0.005$ for Kruskal-Wallis test).

Table 2. Median plasma concentrations (range) and metabolic ratios of risperidone (RIS) in the study groups.

\begin{tabular}{lllll}
\hline Group & RIS & $9-0 H-R I S$ & RIS+9-OH-RIS & $9-0 H-R I S / R I S$ \\
\hline Amlodipine $\left(R_{A}\right)$ & $5.6(0.3-67.0)$ & $19.00(4.5-49.0)$ & $30.50(6.7-112.0)$ & $2.00(0.23-56.67)$ \\
Metoprolol $\left(R_{M}\right)$ & $6.7(0.5-54.0)$ & $17.00(2.0-48.0)$ & $26.00(2.8-82.0)$ & $2.90(0.04-31.0)$ \\
Control $\left(R_{0}\right)$ & $4.4(0.1-224.0)$ & $17.00(0.3-196.5)$ & $24.0(1.8-264.0)$ & $3.8(0.04-290.0)$ \\
\hline
\end{tabular}

9-OH-RIS: 9-hydroxyrisperidone.

metoprolol $\left(\mathrm{R}_{\mathrm{M}}\right)$. No matching processes for age, diagnoses, severity of illness, length or onset of illness were undertaken, and information on trough value or steady-state conditions were lacking and could therefore not be considered in the study. Moreover, patients under concomitant medication with possible CYP2D6 inhibitory or CYP3A4 inhibitory or inducing properties were also excluded. Samples with missing data of RIS or its metabolite were also not included in the analysis.

\section{Statistical analysis}

The analysis included mainly the comparison of three study groups; a group receiving RIS without CYP enzyme influencing co-medication (control group, $\mathrm{R}_{0}$ ), a group receiving RIS + metoprolol $\left(\mathrm{R}_{\mathrm{M}}\right)$ and a group receiving RIS + amlodipine $\left(\mathrm{R}_{\mathrm{A}}\right)$. We compared the medians and the distributions of the plasma concentration of RIS, 9-OH-RIS and the AM (RIS+9-OH-RIS) between the groups. Further comparisons included the plasma concentration corrected by the daily dose, the so called 'concentrationto-dose ratio', (C/D), and the ratios of 9-OH-RIS/RIS for identification of the metabolizer status. Both were calculated in accordance with the AGNP consensus guidelines (Hiemke et al., 2011). Kolmogorov-Smirnov and the Shapiro-Wilk tests of normality indicated that the data were not normally distributed. Therefore, the non-parametrical Kruskal-Wallis test (K-W) with a significance level of 0.05 was conducted. For each comparison of the two different groups $\left(\mathrm{R}_{\mathrm{M}}, \mathrm{R}_{\mathrm{A}}\right)$ with the control group $\left(\mathrm{R}_{0}\right)$ we used the Mann Whitney $U$ test with the same significance level. Finally, the Jonckheere-Terpstra test (J-T) was used to further assess the different patterns of distribution of the adjusted C/D. Statistical analysis was carried out using IBM SPSS Statistics version 18.0 (IBM GmbH, Ehningen, Germany).

\section{Results}

After exclusion of potentially confounding co-medications, 920 out of 1584 patients met the inclusion criteria. Data from these patients were included in the analysis and were assigned to the three groups, $R_{A}, R_{M}$, and $R_{0}$. The demographic data of these patients are summarized in Table 1 . The amlodipine group consists of 27 patients, the metoprolol group of 41 patients, while the control group consists of 852 patients.

We conducted comparisons based upon the K-W with a nominal significance $p<0.05$. The $\mathrm{K}-\mathrm{W}$ detected no differences regarding the median daily dosage of RIS (Table 1) between the three groups ( $p=0.708)$; the mean daily doses for RIS were 4.34 $\mathrm{mg} / \mathrm{d}$, standard deviation $(\mathrm{SD})=2.03$ in the $\mathrm{R}_{0}$-group, $4.13 \mathrm{mg} / \mathrm{d}$, $\mathrm{SD}=2.39$ for $\mathrm{R}_{\mathrm{M}}$ and $3.9 \mathrm{mg} / \mathrm{d}, \mathrm{SD}=2.07$ for $\mathrm{R}_{\mathrm{A}}$. Age and gender distributions showed significant differences between groups $(p<0.001$ and $p=0.005)$; patients under co-medication with amlodipine were older than patients under co-medication with metoprolol and control group patients. Furthermore, there were more women in the $\mathrm{R}_{\mathrm{A}}$-group than in the $\mathrm{R}_{0}$ and the $\mathrm{R}_{\mathrm{M}}$ (see Table 1). The comparison of the distribution (subscript indices 'D') and the medians (subscript indices ' $M$ ') of the plasma concentrations of RIS, 9-OH-RIS, and the AM (RIS+9-OH-RIS) between the three groups did not yield any significant differences $\left(\right.$ RIS $_{\mathrm{D}}, p=0.062,9-0 \mathrm{H}-\mathrm{RIS}_{\mathrm{D}}, p=0.698$ and RIS $+9-\mathrm{OH}-$ $\left.\mathrm{RIS}_{\mathrm{D}}, p=0.369\right) ; \mathrm{RIS}_{\mathrm{M}}\left(p=0.191 ; 9-0 \mathrm{H}-\mathrm{RIS}_{\mathrm{M}}, p=0.898\right.$ and RIS+9-OH-RIS ${ }_{\mathrm{M}}, p=0.266$ ).

The median plasma concentrations $(\mathrm{ng} / \mathrm{mL})$ of RIS, 9-OH-RIS, the AM (RIS+9-OH-RIS), as well as the metabolic ratios (9-OH-RIS/RIS) are displayed in Table 2.

The metabolic ratio (9-OH-RIS/RIS) and the adjusted C/D for the active metabolite 9-OH-RIS did not differ between the groups (9-OH-RIS/RIS $, \quad p=0.059, \quad 9-\mathrm{OH}-\mathrm{RIS} / \mathrm{RIS}_{\mathrm{M}}, \quad p=0.124 ; \quad \mathrm{C} / \mathrm{D}$ 9-OH-RIS $\left.{ }_{\mathrm{D}}, p=0.125, \mathrm{C} / \mathrm{D} 9-\mathrm{OH}-\mathrm{RIS}_{\mathrm{M}}, p=0.208\right)$.

Table 3 shows the C/Ds (ng/mL/mg), for RIS, 9-OH-RIS and RIS+9-OH-RIS for each of the three groups.

The distribution of C/Ds for RIS and for the AM (RIS+9-OHRIS) showed significant differences between the three study groups ( $p=0.006$ and $p=0.005$ respectively) as well as the medians of $\mathrm{C} / \mathrm{D}$ for RIS and RIS+9-OH-RIS ( $p=0.040$ and $p=0.041$ ) (Figure 1).

Using the J-T to detect the different patterns of distribution of the latter parameters (C/D (RIS) and C/D (RIS+9-OH-RIS)), we 
Table 3. Median concentration-to-dose ratios (C/Ds) of risperidone (RIS) in the different groups.

\begin{tabular}{llrr}
\hline Group & \multicolumn{1}{c}{ C/D RIS } & C/D 9-OH-RIS & C/D RIS+9-0H-RIS \\
\hline Amlodipine $\left(R_{A}\right)$ & $2.27 \uparrow^{\mathrm{a}}(0.15-16.75)$ & $5.16 \uparrow^{\mathrm{a}}(1.2-12.25)$ & $9.12 \uparrow^{\mathrm{a}}(2.0-28.0)$ \\
Metoprolol $\left(R_{M}\right)$ & $1.93 \uparrow^{\mathrm{b}}(0.12-27.0)$ & $4.35(0.58-16.0)$ & $7.25(0.7-28.0)$ \\
Control $\left(R_{0}\right)$ & $1.16(0.02-74.67)$ & $4.33(0.08-42.0)$ & $6.29(0.5-88.0)$ \\
\hline
\end{tabular}

9-OH-RIS: 9-hydroxyrisperidone.

${ }^{a} \mathrm{C} / \mathrm{D}$ values for RIS, $9-\mathrm{OH}-\mathrm{RIS}$ and RIS+9-0H-RIS in the $\mathrm{R}_{\mathrm{A}}$ group were significantly higher than in the control group $(p=0.025, p=0.048$ and $p=0.005$ for Mann-Whitney $U$ Test).

${ }^{b} \mathrm{C} / \mathrm{D}$ values for RIS in the RM group were significantly higher than in the control group ( $p=0.017$ for Mann-Whitney $U$ Test).

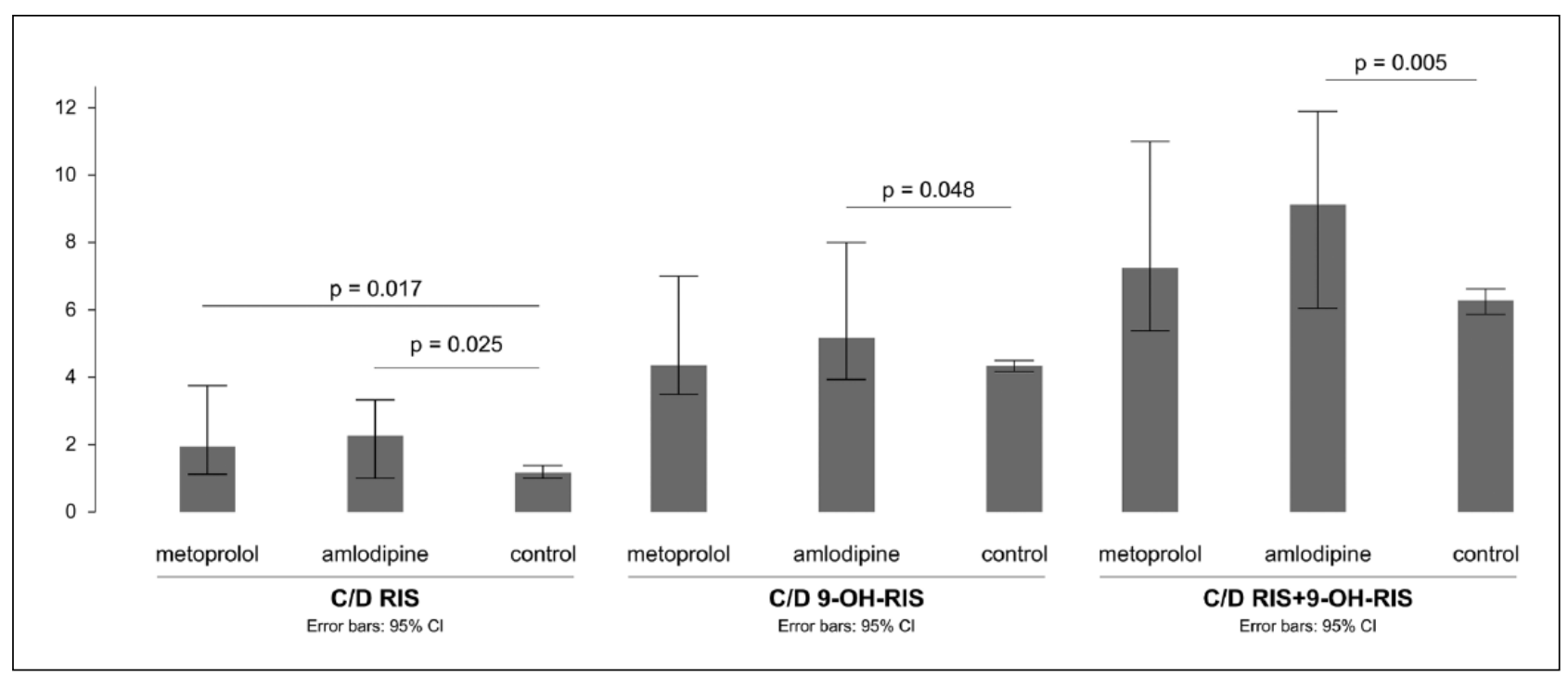

Figure 1. Median concentration-to-dose ratios (C/D) of risperidone (RIS), 9-hydroxyrisperidone (9-OH-RIS) and active moiety in the different groups. CI: confidence interval.

Table 4. Distribution of frequencies of concentration-to-dose ratios (C/Ds) of risperidone (RIS) and active moiety in the different study groups.

\begin{tabular}{llllr}
\hline & & Control & Metoprolol & Amlodipine \\
\hline C/D & $>$ Median & 416 & 26 & 18 \\
RIS & SMedian & 436 & 15 & 9 \\
C/D & $>$ Median & 413 & 24 & 19 \\
RIS +9-OH-RIS & $\leqslant$ Median & 439 & 17 & 8 \\
\hline
\end{tabular}

9-OH-RIS: 9-hydroxyrisperidone.

found an ascending trend for the medians of C/D (RIS) and C/D (RIS+9-OH-RIS) between $\mathrm{R}_{0}, \mathrm{R}_{\mathrm{M}}$ and $\mathrm{R}_{\mathrm{A}}$ (standard (std) J-T value=3.22, $p=0.001$ and 3.086, $p=0.002$ ), with $\mathrm{R}_{0}$ showing the lowest and $\mathrm{R}_{\mathrm{A}}$ showing the highest values.

A Mann-Whitney $U$ test was used to control for significant distribution differences between pairs of groups $\left(R_{0} v s R_{A}\right.$ and $R_{0}$ vs $\left.R_{M}\right) \cdot R_{0}$ vs $R_{A}$ revealed significant distribution differences for C/D RIS $(p=0.025), \mathrm{C} / \mathrm{D}$ 9-OH-RIS $(p=0.048)$ and C/D AM $(p=0.005)$, with the amlodipine group showing higher values in all cases. The metabolic ratios did not differ between $\mathrm{R}_{0}$ and $\mathrm{R}_{\mathrm{A}}$ $(p=0.182)$.

Comparing $\mathrm{R}_{0}$ vs $\mathrm{R}_{\mathrm{M}}$ yielded no differences regarding the distribution of $\mathrm{C} / \mathrm{D}$ for $9-\mathrm{OH}-\mathrm{RIS}$ and $\mathrm{C} / \mathrm{D}$ for AM $(p=0.57$ and $p=0.087$ ) while the distribution of C/D RIS significantly differed between the two groups ( $p=0.017)$, with higher values in the metoprolol group. The distribution of the metabolic ratios showed a slightly significant difference $(p=0.044)$, with the metoprolol group demonstrating significantly lower values.

The distribution differences of C/D RIS and C/D AM between the study groups are quintessentially captured in Table 4 . In the amlodipine group, the number of patients with a 'higher than the median' $\mathrm{C} / \mathrm{D}$, for the AM, RIS+9-OH-RIS, $n=19$, was more than twice as much as the number of patients having a lower than the median $\mathrm{C} / \mathrm{D}(n=8)$. The median $\mathrm{C} / \mathrm{D}$ for the $\mathrm{AM}$ was significantly higher in the amlodipine group $(p=0.041)$. The $\mathrm{C} / \mathrm{D}$ levels for RIS showed a similar pattern in the amlodipine group, so that patients with a 'higher than the median' C/D (RIS) were twice $(n=18)$ as many as the ones with 'lower than the median' C/D 
levels ( $n=9$ ). It should be noted that the C/D (RIS) was significantly higher in the amlodipine group ( $p=0.04)$.

\section{Discussion}

Cardiovascular diseases including arterial hypertension are highly prevalent in patients with severe mental disorders such as schizophrenia and the pharmacological treatment may be essential in eliminating the excess mortality rate and improving life expectancy (Crump et al., 2013; Ringen et al., 2014). By adding antihypertensive and/or cardioprotective drugs to an ongoing antipsychotic treatment, the risk of pharmacokinetic DDIs is increasing. To predict potential DDIs, knowledge about potential CYP450 mediated interactions as one source of an increased risk is essential to enhance safety and tolerability in the treatment of the psychiatric disease as well as in the treatment of the somatic disease. Knowledge about metabolic pathways of concomitantly applied drugs and the potential of any particular drug to induce or inhibit CYP isoenzymes is of high clinical importance to prevent the patient from unwanted side effects or to prevent the patient from loss of treatment efficacy. Data from TDM surveys provide an essential source to better understand the effects of polypharmacy in clinical settings (Schoretsanitis et al., 2016). The primary aim of this study was to compare the potential of two co-medications, amlodipine and metoprolol, to inhibit CYP2D6 and/or CYP3A4/5 and thereby influence the metabolism of an antipsychotic treatment with RIS which is mainly but not exclusively metabolized via CYP2D6.

To our knowledge, information about potential pharmacokinetic DDIs between amlodipine and RIS as well as between metoprolol and RIS is lacking thus far and our findings support the notion that the selection of the optimal antihypertensive drugs should not only take psychiatric comorbidities into account but also concomitant psychotropic medication.

In our naturalistic sample, patients on a stable dose of RIS in the control group and patients who were co-medicated with amlodipine or metoprolol demonstrated considerably different patterns of plasma concentrations of RIS, 9-OH-RIS and AM as well as different patterns of C/Ds. While the daily dosage of RIS did not differ significantly between the study groups, patients that were co-medicated with amlodipine showed significantly higher median values for the $\mathrm{C} / \mathrm{Ds}$ of the parent compound, C/D RIS, the active metabolite, C/D 9-OH-RIS and the AM, C/D (RIS+9-OHRIS), than patients in the control group. Patients that were comedicated with metoprolol showed significantly higher median values for C/D RIS while C/D 9-OH-RIS and C/D for the AM did not differ from the control group. Furthermore, the distribution of the concentration-to-dose ratios showed an interesting pattern in the sense that patients in the amlodipine group were twice as likely to show C/D levels for RIS and the AM above the median as controls. Although pharmacokinetic parameters showed a high inter-individual variability, which has been detected in other studies as well (Balant-Gorgia et al., 1999; Cabaleiro et al., 2015; Feng et al., 2008), it should be noted that we observed no additional distribution differences regarding plasma concentrations and metabolic ratios of RIS as well as the C/Ds of the active metabolite of RIS (9-OH-RIS) between the groups. To our understanding, the findings presented herein imply a potential inhibiting effect of amlodipine most likely on the CYP3A4/5 mediated metabolism of RIS, leading to significantly higher median values of the concentration-to-dose ratios of RIS, 9-OH-RIS and the AM.
This hypothesis might be supported by the fact that amlodipine is metabolized via CYP3A4 on the one hand and is known to have inhibitory effects on CYP3A4 as well. The latter properties have been shown to have clinical relevance for plasma concentrations of the HMG-CoA reductase inhibitor simvastatin which is used to provide cholesterol-lowering effects (Nishio et al., 2005; Son et al., 2014). Consequentially, we suggest a moderate inhibiting effect of amlodipine on CYP3A4 leading to significantly different concentration-to-dose ratios but without significant effects on the plasma concentration of RIS and its active metabolite or the AM. This effect can have potential clinical implications with regard to clinical response and adverse effects (De Leon et al., 2005; Riedel et al., 2005), particularly since antipsychotic polypharmacy is prevalent (Gallego et al., 2012; Spina and de Leon, 2007) and in case of RIS, since 9-OH-RIS is eliminated more slowly which can be seen in a longer half-life time and a lower plasma protein binding than RIS.

As different CYP isoenzymes are involved in the metabolism of RIS to its renally excreted active metabolite 9-OH-RIS, the pharmacokinetic interaction potential of concomitantly applied drugs not only concerning their ability to influence the main metabolic pathway via CYP2D6 have to be considered. Hence, amlodipine with its impact on downstream metabolic pathways of RIS via CYP3A4 is an important example for potential pharmacokinetic interactions offside the main metabolic pathway.

The co-medication with metoprolol slightly altered the concentration of C/D RIS compared with the control group. Patients under metoprolol had higher C/D RIS values than the control group. However, this increase was not reflected in the $\mathrm{C} / \mathrm{D}$ for the AM, showing no significant difference between the two groups. This illustrates a rather slight inhibitory effect of metoprolol on RIS metabolism, which was further implied by the lower metabolic ratio in the metoprolol group ( $p=0.044)$. This finding should be considered in the light of the common metabolic pathway of RIS and metoprolol, since they're both metabolized by CYP2D6. To our knowledge, no data have been reported suggesting an auto-inhibiting effect of metoprolol. Consequently, one might assume a weak effect of metoprolol on secondary metabolic pathways of RIS like CYP3A4, which can lead to a slight increase of $\mathrm{C} / \mathrm{D}$ of parent compound and/or a competitive effect as both drugs are metabolized via the same pathway.

Based upon this data and from a pharmacokinetic point of view, it should be taken into account when prescribing amlodipine to an ongoing treatment with RIS that the addition leads to changes in $\mathrm{C} /$ Ds. This insight might reveal a comparative disadvantage when amlodipine is used as a co-medication. Calcium-channel blockers are widely assumed to be a safe and very well tolerable option for initial antihypertensive therapy (James et al., 2014); however, potential interactions, presumably mediated by the CYP450 pathway of drug metabolism, have to be considered in order to minimize adverse and/or unwanted effects. Theoretically, beta-blockers could be a more favourable choice for treating arterial hypertension in patients with schizophrenia, taking into account a possible underlying adrenergic hyperactivity (Scigliano and Ronchetti, 2013). Even though we examined only one agent of this pharmacological class, the focus should be placed on the rather disadvantageous metabolic profile of other compounds such as metoprolol which, compared to other beta-blockers, has been shown to decrease insulin sensitivity (Ayers et al., 2012; Bakris et al., 2004; Phillips et al., 2008). Taking into account the well-known risk 
factors of patients with severe mental illnesses, this could be a less favourable option for the optimal antihypertensive therapy regimen.

\section{Limitations}

Our sample comprised a large population of naturalistic nature and relies on retrospective data. A significant amount of clinical parameters (onset and duration of illness, adverse effects, comorbidities, duration of drug exposure) were not available. Furthermore, there is a large individual variation in sampling time as a result of the clinical setting, which may have partially accounted for the pronounced inter-individual variation in plasma concentrations and metabolic ratios. Besides this large interindividual variability in RIS and 9-OH RIS concentrations has been already reported in the literature during routine TDM (Balant-Gorgia et al., 1999). In the case of multiple plasma concentration determinations we minimized the patient bias by including only one analysis per patient. Regarding analyses of the data, differences in sample characteristics and sample size in the three patient groups occurred; thus the comparability of the study groups with the control group might be restricted. However, in some cases, e.g. regarding the mean age of the different study groups, it appeared plausible that patients under antihypertensive therapy would be older. Consequently, we avoided stratifying for age despite the well-known effect of age on RIS metabolism (Feng et al., 2008). Although the size of the control group is remarkably bigger, we chose not to reduce our control group taking into account the extent of the skewness of the sample distribution.

\section{Acknowledgements}

Authors who participated in research design were MP, GS, GG, EH, BS, KRJS, SW; performed data analysis: GS, MP, SEL; wrote or contributed to the writing of the manuscript: MP, GS, GG, SW, EH, BS, KRJS, SEL. The authors wish to express their appreciation to Lisa Deuse for carefully reading the manuscript.

\section{Declaration of Conflicting Interests}

The authors declared the following potential conflicts of interest with respect to the research, authorship, and/or publication of this article:

Ekkehard Haen received speaker or consultancy fees from the following pharmaceutical companies: Servier, Novartis and Janssen-Cilag. $\mathrm{He}$ is managing director of AGATE, a non-profit working group to improve drug safety and efficacy in the treatment of psychiatric diseases. $\mathrm{He}$ reports no conflict of interest with this publication.

Gerhard Gründer has served as a consultant for Boehringer Ingelheim (Ingelheim, Germany), Cheplapharm (Greifswald, Germany), Eli Lilly (Indianapolis, Indiana, USA), Lundbeck (Copenhagen, Denmark), Ono Pharmaceuticals (Osaka, Japan), Roche (Basel, Switzerland), Servier (Paris, France) and Takeda (Osaka, Japan). He has served on the speakers' bureau of Eli Lilly, Gedeon Richter (Budapest, Ungarn), Janssen Cilag (Neuss, Germany), Lundbeck, Roche, Servier and Trommsdorf (Aachen, Germany). He has received grant support from Boehringer Ingelheim and Roche. He is co-founder of Pharma Image $\mathrm{GmbH}$ (Düsseldorf, Germany) and Brainfoods UG (Selfkant, Germany). He reports no conflict of interest with this publication.

Georgios Schoretsanitis received a grant from the bequest 'in memory of Maria Zaoussi', State Scholarships Foundation, Greece for clinical research in psychiatry for the academic year 2015-2016.

MP, SEL, BS, KRJS and SW declared no potential conflicts of interest with respect to the research, authorship, and/or publication of this article.

\section{Funding}

The authors received no financial support for the research, authorship, and/or publication of this article.

\section{References}

Ayers K, Byrne LM, DeMatteo A, et al. (2012) Differential effects of nebivolol and metoprolol on insulin sensitivity and plasminogen activator inhibitor in the metabolic syndrome. Hypertension 59: 893-898.

Bakris GL, Fonseca V, Katholi RE, et al. (2004) Metabolic effects of carvedilol vs metoprolol in patients with type 2 diabetes mellitus and hypertension: A randomized controlled trial. JAMA 292: 2227-2236.

Balant-Gorgia AE, Gex-Fabry M, Genet C, et al. (1999) Therapeutic drug monitoring of risperidone using a new, rapid HPLC method: Reappraisal of interindividual variability factors. Ther Drug Monit 21: 105-115.

Bresee LC, Majumdar SR, Patten SB, et al. (2010) Prevalence of cardiovascular risk factors and disease in people with schizophrenia: A population-based study. Schizophr Res 117: 75-82.

Cabaleiro T, Ochoa D, Roman M, et al. (2015) Polymorphisms in CYP2D6 have a greater effect on variability of risperidone pharmacokinetics than gender. Basic Clin Pharmacol Toxicol 116: 124-128.

Cadieux RJ (1989) Drug interactions in the elderly. How multiple drug use increases risk exponentially. Postgrad Med 86: 179-186.

Chouinard G and Arnott W (1993) Clinical review of risperidone. Can J Psychiatry 38: S89-S95.

Correll CU, Lencz T and Malhotra AK (2011) Antipsychotic drugs and obesity. Trends Mol Med 17: 97-107.

Crump C, Winkleby MA, Sundquist K, et al. (2013) Comorbidities and mortality in persons with schizophrenia: A Swedish national cohort study. Am J Psychiatry 170: 324-333.

De Leon J, Susce MT, Pan RM, et al. (2005) The CYP2D6 poor metabolizer phenotype may be associated with risperidone adverse drug reactions and discontinuation. $J$ Clin Psychiatry 66: 15-27.

Fang J, Bourin M and Baker GB (1999) Metabolism of risperidone to 9-hydroxyrisperidone by human cytochromes P450 2D6 and 3A4. Naunyn-Schmiedeberg's Arch Pharmacol 359: 147-151.

Faulkner JK, McGibney D, Chasseaud LF, et al. (1986) The pharmacokinetics of amlodipine in healthy volunteers after single intravenous and oral doses and after 14 repeated oral doses given once daily. $\mathrm{Br}$ J Clin Pharmacol 22: 21-25.

Feng Y, Pollock BG, Coley K, et al. (2008) Population pharmacokinetic analysis for risperidone using highly sparse sampling measurements from the CATIE study. Br J Clin Pharmacol 66: 629-639.

Fervaha G, Foussias G, Agid O, et al. (2014) Impact of primary negative symptoms on functional outcomes in schizophrenia. Eur Psychiatry 29: 449-455.

Fukao K, Shimada K, Hiki M, et al. (2011) Effects of calcium channel blockers on glucose tolerance, inflammatory state, and circulating progenitor cells in non-diabetic patients with essential hypertension: A comparative study between azelnidipine and amlodipine on glucose tolerance and endothelial function-a crossover trial (AGENT). Cardiovasc Diabetol 10: 79. DOI: 10.1186/1475-2840-10-79.

Gallego JA, Bonetti J, Zhang J, et al. (2012) Prevalence and correlates of antipsychotic polypharmacy: A systematic review and meta-regression of global and regional trends from the 1970s to 2009. Schizophr Res 138: 18-28.

Goff DC, Sullivan LM, McEvoy JP, et al. (2005) A comparison of tenyear cardiac risk estimates in schizophrenia patients from the CATIE study and matched controls. Schizophr Res 80: 45-53.

Hefner G, Unterecker S, Shams ME, et al. (2015) Melperone but not bisoprolol or metoprolol is a clinically relevant inhibitor of CYP2D6: Evidence from a therapeutic drug monitoring survey. J Neural Transm 122: 1609-1617.

Hennekens CH, Hennekens AR, Hollar D, et al. (2005) Schizophrenia and increased risks of cardiovascular disease. Am Heart $J 150$ : 1115-1121. 
Hiemke C, Baumann P, Bergemann N, et al. (2011) AGNP Consensus guidelines for therapeutic drug monitoring in psychiatry: Update 2011. Pharmacopsychiatry 44: 195-235.

James PA, Oparil S, Carter BL, et al. (2014) 2014 evidence-based guideline for the management of high blood pressure in adults: Report from the panel members appointed to the Eighth Joint National Committee (JNC 8). JAMA 311: 507-520.

Janssen PA, Niemegeers CJ, Awouters F, et al. (1988) Pharmacology of risperidone ( $\mathrm{R} 64$ 766), a new antipsychotic with serotonin-S2 and dopamine-D2 antagonistic properties. J Pharmacol Exp Ther 244: 685-693.

Kim KA, Park PW, Lee OJ, et al. (2006) Effect of CYP3A5*3 genotype on the pharmacokinetics and pharmacodynamics of amlodipine in healthy Korean subjects. Clin Pharmacol Ther 80: 646-656.

Kjeldsen S, Feldman RD, Lisheng L, et al. (2014) Updated national and international hypertension guidelines: A review of current recommendations. Drugs 74: 2033-2051.

Lee SA, Choi HM, Park HJ, et al. (2014) Amlodipine and cardiovascular outcomes in hypertensive patients: Meta-analysis comparing amlodipine-based versus other antihypertensive therapy. Korean $J$ Intern Med 29: 315-324.

Leucht S, Pitschel-Walz G, Abraham D, et al. (1999) Efficacy and extrapyramidal side-effects of the new antipsychotics olanzapine, quetiapine, risperidone, and sertindole compared to conventional antipsychotics and placebo. A meta-analysis of randomized controlled trials. Schizophr Res 35: 51-68.

Li Q and Wang R (2006) Simultaneous analysis of tramadol, metoprolol and their metabolites in human plasma and urine by high performance liquid chromatography. Chin Med J (Engl) 119: 2013-2017.

Liao CH, Chang CS, Wei WC, et al. (2011) Schizophrenia patients at higher risk of diabetes, hypertension and hyperlipidemia: A population-based study. Schizophr Res 126: 110-116.

Marder SR, Davis JM and Chouinard G (1997) The effects of risperidone on the five dimensions of schizophrenia derived by factor analysis: Combined results of the North American trials. J Clin Psychiatry 58: 538-546.

Nasrallah HA, Harvey PD, Casey D, et al. (2015) The Management of Schizophrenia in Clinical Practice (MOSAIC) Registry: A focus on patients, caregivers, illness severity, functional status, disease burden and healthcare utilization. Schizophr Res 166: 69-79.

Nasrallah HA, Meyer JM, Goff DC, et al. (2006) Low rates of treatment for hypertension, dyslipidemia and diabetes in schizophrenia: Data from the CATIE schizophrenia trial sample at baseline. Schizophr Res 86: 15-22.

Nishio S, Watanabe H, Kosuge K, et al. (2005) Interaction between amlodipine and simvastatin in patients with hypercholesterolemia and hypertension. Hypertens Res 28: 223-227.
Phillips RA, Fonseca V, Katholi RE, et al. (2008) Demographic analyses of the effects of carvedilol vs metoprolol on glycemic control and insulin sensitivity in patients with type 2 diabetes and hypertension in the Glycemic Effects in Diabetes Mellitus: Carvedilol-Metoprolol Comparison in Hypertensives (GEMINI) study. J Cardiometab Syndr 3: 211-217.

Rabinowitz J, Levine SZ, Garibaldi G, et al. (2012) Negative symptoms have greater impact on functioning than positive symptoms in schizophrenia: Analysis of CATIE data. Schizophr Res 137: 147-150.

Riedel M, Schwarz MJ, Strassnig M, et al. (2005) Risperidone plasma levels, clinical response and side-effects. Eur Arch Psychiatry Clin Neurosci 255: 261-268.

Ringen PA, Engh JA, Birkenaes AB, et al. (2014) Increased mortality in schizophrenia due to cardiovascular disease - a non-systematic review of epidemiology, possible causes, and interventions. Front Psychiatry 5: 137. DOI: 10.3389/fpsyt.2014.00137.

Schoretsanitis G, Haen E, Hiemke H, et al. (2016) Risperidone induced extrapyramidal side effects: Is the need for anticholinergics the consequence of high plasma concentrations? Int Clin Psychopharmacol [Epub ahead of print] 10 May 2016. DOI: 10.1097/ YIC.0000000000000131

Scigliano G and Ronchetti G (2013) Antipsychotic-induced metabolic and cardiovascular side effects in schizophrenia: A novel mechanistic hypothesis. CNS Drugs 27: 249-257.

Sicras-Mainar A, Maurino J, Ruiz-Beato E, et al. (2015) Prevalence of metabolic syndrome according to the presence of negative symptoms in patients with schizophrenia. Neuropsychiatr Dis Treat 11: 51-57.

Son H, Lee D, Lim LA, et al. (2014) Development of a pharmacokinetic interaction model for co-administration of simvastatin and amlodipine. Drug Metab Pharmacokinet 29: 120-128.

Spina E and de Leon J. (2007) Metabolic drug interactions with newer antipsychotics: A comparative review. Basic Clin Pharmacol Toxicol 100: 4-22.

Stingl JC, Brockmoller J and Viviani R (2013) Genetic variability of drug-metabolizing enzymes: The dual impact on psychiatric therapy and regulation of brain function. Mol Psychiatry 2013; 18: 273-287.

US Food and Drug Administration (2014) Drug development and drug interactions: Table of substrates, inhibitors and inducers, http:// www.fda.gov/Drugs/DevelopmentApprovalProcess/DevelopmentResources/DrugInteractionsLabeling/ucm093664.htm (accessed 10 April 2016).

Xiang Q, Zhao X, Zhou Y, et al. (2010) Effect of CYP2D6, CYP3A5, and MDR1 genetic polymorphisms on the pharmacokinetics of risperidone and its active moiety. J Clin Pharmacol 50: 659-666.

Yasui-Furukori N, Hidestrand M, Spina E, et al. (2001) Different enantioselective 9-hydroxylation of risperidone by the two human CYP2D6 and CYP3A4 enzymes. Drug Metab Dispos 29: 1263-1268. 